\title{
Evaluation of disk halo size after small incision lenticule extraction (SMILE)
}

\author{
Tian Han ${ }^{1,2,3} \cdot$ Feng Zhao ${ }^{1,2,3,4} \cdot$ Xun Chen $^{1,2,3} \cdot$ Huamao Miao ${ }^{1,2,3} \cdot$ Zhuoyi Chen $^{1,2,3} \cdot$ Xingtao Zhou $^{1,2,3} \mathbb{C}$
}

Received: 4 June 2019 / Revised: 21 August 2019 / Accepted: 13 September 2019 / Published online: 29 October 2019

(C) The Author(s) 2019

\begin{abstract}
Purpose To investigate changes in objective disk halo size produced by a glare source after small incision lenticule extraction (SMILE) for myopia correction.

Methods This prospective clinical study included 45 right eyes of 45 patients with a mean age of $25.40 \pm 5.06$ years and mean spherical equivalent (SE) of $-6.08 \pm 1.90$ diopters. Disk halo size was measured with a vision monitor before surgery and at postoperative 1 week and 3 months. Other information was collected, including age, SE, lenticule thickness, lenticule diameter, dark pupil, and pupillary response to light parameters (initial diameter; amplitude, latency, duration, and velocity of contraction; latency, duration, and velocity of dilation; and maximum, minimum, and average pupil size).

Results Compared to preoperative values, disk halo size increased significantly at postoperative 1 week $(P=0.026)$ and returned to baseline at postoperative 3 months $(P=0.349)$. Preoperative disk halo size significantly correlated with SE $(r=-0.346, P=$ $0.020)$, minimum pupil size $(r=0.365, P=0.014)$, and average pupil size $(r=0.310, P=0.038)$. Disk halo size at postoperative 1 week was significantly correlated with age $(r=0.324, P=0.030)$ and minimum pupil size $(r=0.297, P=0.047)$. Disk halo size at postoperative 3 months was significantly correlated with lenticule diameter $(r=-0.362, P=0.015)$, initial diameter $(r=0.311$, $P=0.037)$, maximum pupil size $(r=0.312, P=0.037)$, minimum pupil size $(r=0.440, P=0.002)$, and average pupil size $(r=$ $0.373, P=0.012$ ).
\end{abstract}

Conclusions After SMILE, disk halo size demonstrated a temporary increase and then returned to baseline.

Keywords Small incision lenticule extraction (SMILE) $\cdot$ Disk halo size $\cdot$ Pupillary light response $\cdot$ Glare

\section{Introduction}

Light from a bright light source entering the retina is diffused by the various optical elements of the eye. The diffused light

Tian Han and Feng Zhao contributed equally to this study and are considered co-first authors.

Xingtao Zhou

doctzhouxingtao@163.com

1 Department of Ophthalmology and Vision Science, The Eye and ENT Hospital of Fudan University, No.19 Baoqing Road, Xuhui District, Shanghai, China

2 NHC Key Laboratory of Myopia (Fudan University); Laboratory of Myopia, Chinese Academy of Medical Sciences, Shanghai, China

3 Research Center of Ophthalmology and Optometry Shanghai, Shanghai, China

4 The Department of Ophthalmology, Shuguang Hospital of Shanghai University of Traditional Chinese Medicine, Shanghai, China creates a veil over the back of the retina which can lead to a loss in contrast and details of an object. The phenomenon is referred to as disability glare, and the veil is known as disk halos [1,2]. Glare and halos are common complaints after refractive surgery that require special attention, especially after corneal refractive surgery. In many patient satisfaction surveys after corneal refractive surgery, glare and halos are the main complaints [3-7].

Small incision lenticule extraction (SMILE) benefits from the well-developed femtosecond laser technology and exhibits many advantages over traditional laser in situ keratomileusis (LASIK). For example, it can avoid the flap-related complications encountered in LASIK surgery and reduce corneal nerve damage, thereby alleviating postoperative dry eye symptoms and maintaining corneal sensitivity [8-10]. Many studies have confirmed the safety and stability of SMILE surgery for myopia correction [11, 12].

Considering that glare and halos remain common problems after SMILE surgery [13], changes in glare and halos after 
SMILE surgery require further studies. Previous investigations have shown changes in glare after SMILE using a double-pass optical quality analysis system (OQAS) [14] or a straylight meter (C-Quant) [15]. To the best of our knowledge, however, no study has focused on halo size measurements in relation to SMILE surgery.

Here, we analyzed correlations between disk halo size produced by a glare source and pupillary light response parameters, in order to understand disk halo size after SMILE and determine its influencing factors.

\section{Methods}

This study was approved by the Ethics Committee of the Eye and ENT Hospital of Fudan University and complied with the Declaration of Helsinki. Informed written consent was obtained from all patients after the possible consequences of the study were explained.

\section{Study population}

Consecutive patients were enrolled in this prospective study. All patients underwent routine preoperative ophthalmic examinations at the Refractive Surgery Center in the Department of Ophthalmology of the Eye and ENT Hospital of Fudan University.

The inclusion criteria were as follows: patients aged between 18 and 35 years, spherical equivalent (SE) up to 9.5 diopters (D), corrected distance visual acuity (CDVA) of $20 / 20$ or better, and stable refraction for 2 years. Because age has relationships with both the pupillary response to light and disk halo size [16], and patients between 20 and 50 years of age have similar disk halo sizes [17], the inclusion age range was modified to $18-35$ years.

The exclusion criteria were as follows: systemic diseases, a history of ocular surgery or trauma, and a history of ocular disease other than myopia or astigmatism.

Data from the right eye of each patient were selected for statistical analysis.

\section{Surgical procedure}

The same surgeon (Dr. Zhou) performed all surgical procedures. In the SMILE procedures, a 500-kHz VisuMax femtosecond laser system (Carl Zeiss Meditec, Jena, Germany) was used, with a pulse energy of $130 \mathrm{~nJ}$. The lenticule diameter was set between 6.25 and $6.70 \mathrm{~mm}$; the cap diameter was set to $7.5 \mathrm{~mm}$ at a $120-\mu \mathrm{m}$ depth. A $90^{\circ}$ single-side cut, with a length of $2 \mathrm{~mm}$, was created to remove the lenticule. Topical levofloxacin, $0.1 \%$ fluorometholone solution, and nonpreserved artificial tears were used after the surgery.

\section{Measurements}

Disk halo size and pupillary light response were evaluated by an experienced technician before surgery, and at postoperative 1 week and 3 months with monocular dynamic pupillometry (MonCv3; Metrovision, Pérenchies, France).

As described in previous studies [17, 18], all measurements were obtained between 9:00 a.m. and 11:30 a.m. after $5 \mathrm{~min}$ of darkness adaptation. A light source on the right side was used to test the right eye with a luminance of $5 \mathrm{~cd} / \mathrm{m}^{2}$. The refractive error was fully corrected with a lens before surgery. Because all patients had worn glasses before SMILE, we presume that our measurements approximate situations in their daily lives. There were three radial lines of 10 letters appearing from the periphery toward the light source on the screen such that 10 letters formed 10 rings at $30-\mathrm{min}$ arc intervals ( $\operatorname{arcmin}$ ). The average distance to the letter nearest to the light source was measured for each line, and then the visual angle formed by the radius of the halo was calculated in arcmin (Fig. 1).

The pupillary contour was automatically traced by the pupillometer with an accuracy of $\pm 0.1 \mathrm{~mm}$ after $5 \mathrm{~min}$ of darkness adaptation. Then, the software performed an analysis of responses to successive visual stimuli with automated quantification of the following parameters: initial diameter; amplitude, latency, duration, and velocity of contraction; latency,

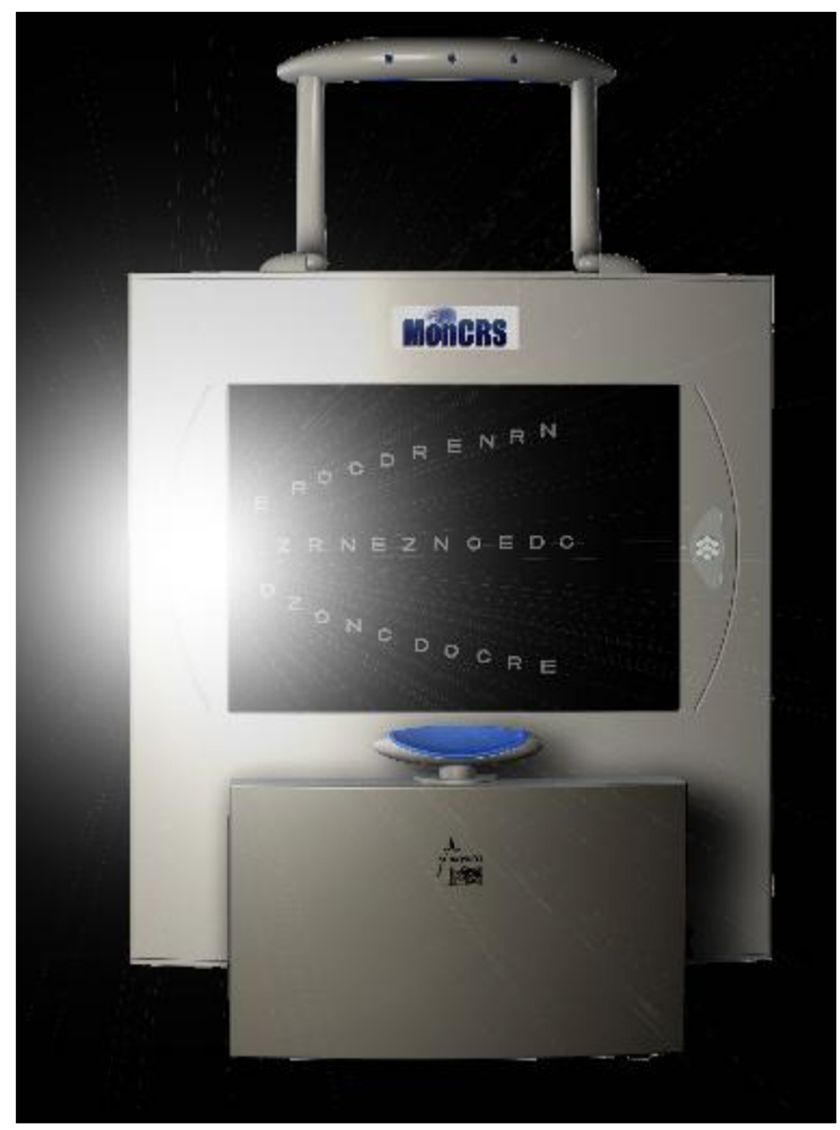

Fig. 1 The operative interface of the vision monitor 
duration, and velocity of dilation; and maximum, minimum, and average pupil sizes. Each parameter was measured at least five times and the mean values were recorded.

\section{Statistical analysis}

All statistical analyses were performed using the Statistical Package for Social Sciences (SPSS) (version 22; IBM, Armonk, NY, USA). All data were tested for normality using the Kolmogorov-Smirnov test. The Friedman test with a Bonferroni correction was performed to evaluate changes in disk halo size over time. Pearson's and Spearman's correlation analyses were applied to detect potential correlations between disk halo size and other parameters. A $P$ value less than 0.05 was considered statistically significant.

\section{Results}

Forty-five patients with a mean age of $25.40 \pm 5.06$ years (range 18 to 35 years) and mean SE of $-6.08 \pm 1.90 \mathrm{D}$ (range -3.50 to $-8.75 \mathrm{D})$ were enrolled. Table 1 shows the demographic and refractive data. All surgeries were uneventful, and no intraoperative or postoperative complications were observed. No patient was lost to follow-up in this study.

Compared to preoperative values $(166.22 \pm 61.29)$, disk halo size increased significantly at postoperative 1 week

Table 1 Demographic and refractive data $(n=45)$

\begin{tabular}{lrr}
\hline Parameters & Mean & \multicolumn{1}{c}{ SD } \\
\hline Age (years) & 25.40 & 5.06 \\
Sphere (D) & -5.60 & 1.82 \\
Cylinder (D) & -0.96 & 0.85 \\
SE (D) & -6.08 & 1.90 \\
Lenticule diameter (mm) & 6.58 & 0.21 \\
Lenticule thickness (um) & 113.13 & 28.96 \\
Dark pupil (mm) & 7.06 & 0.65 \\
Initial diameter (mm) & 5.33 & 0.64 \\
Amplitude of contraction (mm) & 1.83 & 0.29 \\
Latency of contraction (ms) & 239.09 & 64.68 \\
Duration of contraction (ms) & 647.09 & 75.87 \\
Velocity of contraction (mm/s) & 5.81 & 0.88 \\
Latency of dilation (ms) & 886.29 & 60.78 \\
Duration of dilation (ms) & 1574.76 & 73.08 \\
Velocity of dilation (mm/s) & 2.34 & 0.48 \\
Maximum pupil (mm) & 5.93 & 0.64 \\
Minimum pupil (mm) & 3.43 & 0.44 \\
Average pupil (mm) & 4.82 & 0.54 \\
\hline
\end{tabular}

$D$ diopters, $S E$ spherical equivalent

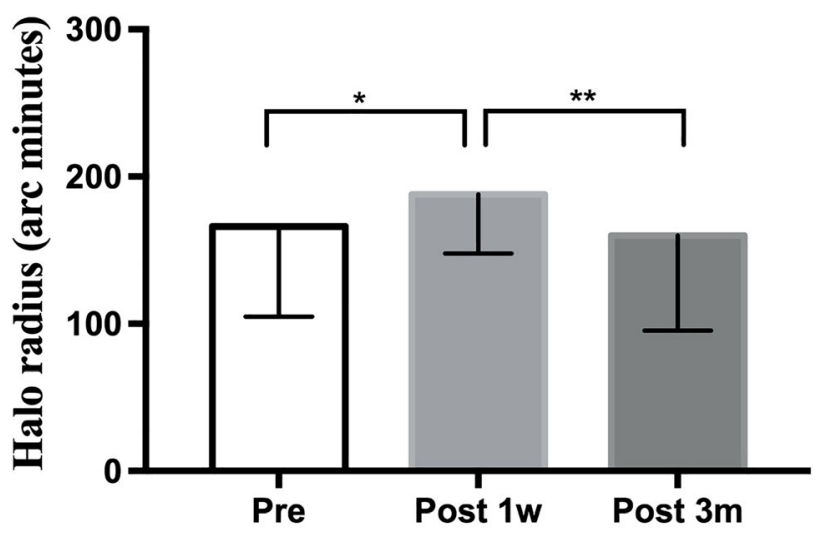

Time course of halo radius after SMILE

Fig. 2 Time course of halo radius after SMILE. $* * P<0.01, * P<0.05$

$(187.78 \pm 39.88)(P=0.026)$ and returned to baseline at postoperative 3 months $(160.22 \pm 64.75)(P=0.349)$ (Fig. 2).

Table 2 shows the correlation analysis between disk halo size and age, spherical equivalent refraction, and pupil parameters. Preoperative disk halo size was significantly correlated with SE $(r=-0.346, P=0.020)$, minimum pupil size $(r=$ $0.365, P=0.014)$, and average pupil size $(r=0.310, P=$ 0.038 ). Disk halo size at postoperative 1 week was significantly correlated with age $(r=0.324, P=0.030)$ and minimum pupil size $(r=0.297, P=0.047)$. Disk halo size at postoperative 3 months was significantly correlated with lenticule diameter $(r=-0.362, P=0.015)$, initial diameter $(r=0.311$, $P=0.037)$, maximum pupil size $(r=0.312, P=0.037)$, minimum pupil size $(r=0.440, P=0.002)$, and average pupil size $(r=0.373, P=0.012)$.

\section{Discussion}

Glare and halos remain an important factor in postoperative patient satisfaction [3-6]. This study was undertaken to investigate early changes in disk halo size produced by a glare source after SMILE.

We found that the disk halo size of patients undergoing SMILE initially increased, then decreased, eventually returning to the preoperative level at 3 months after surgery; this is consistent with results obtained by using other glare testing instruments. A study that used OQAS to observe changes in objective scatter index after SMILE found that, within 40 days after the surgery, the index was higher than preoperative levels, but recovered by 3 months after the surgery [14]. No significant difference was found in subjective intraocular forward scattering, assessed by using C-Quant before surgery, and at 1, 6, and 12 months after SMILE [15]. The results of our study are also consistent with subjective questionnaire studies on halos. In a study by Shah et al., no 
Table 2 Correlation analysis between age, spherical equivalent refraction, pupil parameters, and halo radius (arc minutes)

\begin{tabular}{|c|c|c|c|c|c|c|}
\hline \multirow[t]{2}{*}{ Variables } & \multicolumn{2}{|c|}{ Preoperation } & \multicolumn{2}{|c|}{ Postoperative 1 week } & \multicolumn{2}{|c|}{ Postoperative 3 months } \\
\hline & $R$ & $P$ & $R$ & $P$ & $R$ & $P$ \\
\hline Age (years) & -0.098 & 0.520 & $0.324^{*}$ & 0.030 & -0.205 & 0.176 \\
\hline Preoperative SE (D) & $-0.346^{*}$ & 0.020 & -0.117 & 0.445 & -0.283 & 0.060 \\
\hline Lenticule thickness (um) & / & / & 0.092 & 0.548 & 0.236 & 0.118 \\
\hline Lenticule diameter (mm) & l & l & -0.242 & 0.109 & $-0.362^{*}$ & 0.015 \\
\hline Dark pupil (mm) & 0.106 & 0.487 & 0.037 & 0.809 & 0.281 & 0.062 \\
\hline Initial diameter (mm) & 0.217 & 0.152 & 0.208 & 0.170 & $0.311^{*}$ & 0.037 \\
\hline Amplitude of contraction (mm) & -0.086 & 0.576 & -0.012 & 0.937 & -0.057 & 0.712 \\
\hline Latency of contraction (ms) & -0.095 & 0.535 & -0.005 & 0.975 & 0.041 & 0.790 \\
\hline Duration of contraction (ms) & 0.019 & 0.904 & -0.009 & 0.955 & -0.106 & 0.487 \\
\hline Velocity of contraction $(\mathrm{mm} / \mathrm{s})$ & -0.139 & 0.363 & -0.027 & 0.859 & -0.130 & 0.396 \\
\hline Latency of dilation (ms) & -0.141 & 0.354 & -0.041 & 0.790 & -0.028 & 0.855 \\
\hline Duration of dilation (ms) & -0.066 & 0.668 & -0.193 & 0.205 & -0.037 & 0.809 \\
\hline Velocity of dilation $(\mathrm{mm} / \mathrm{s})$ & 0.192 & 0.207 & 0.024 & 0.875 & 0.149 & 0.328 \\
\hline Maximum pupil (mm) & 0.227 & 0.133 & 0.150 & 0.325 & $0.312^{*}$ & 0.037 \\
\hline Minimum pupil (mm) & $0.365^{*}$ & 0.014 & $0.297^{*}$ & 0.047 & $0.440^{*}$ & 0.002 \\
\hline Average pupil (mm) & $0.310^{*}$ & 0.038 & 0.263 & 0.081 & $0.373^{*}$ & 0.012 \\
\hline
\end{tabular}

$S E$ spherical equivalent, $D$ diopters, $R$ correlation coefficient; $* P<0.05$ deterioration in halos was reported by patients at postoperative 3 months, compared with preoperative levels of halos [19]. The time we observed for recovery from increased postoperative halos was similar to that in questionnaire studies after LASIK. At postoperative 3 months, halo scores were indistinguishable from baseline in a study by Schallhorn et al. [20] In addition, corneal transparency decreases for a short period of time after SMILE surgery and recovers at the later stage, which may explain the short-term increase observed in this study [21].

In this study, we found that the disk halo size in myopic patients before SMILE was correlated with SE, which is consistent with our previous studies [22]. Preoperative disk halo size was $166.22 \pm 61.29$ arcmin; this was notably different from $111.6 \pm 39.8$ arcmin in healthy eyes with limited refractive error [17], and was similar to other outcomes in myopic eyes [22]. Short-term disk halo size after SMILE is associated with age. This may explain lower comparative satisfaction with corneal refractive surgery in older patients [7].

At 3 months after surgery, the corneal condition in the patients became stable. Disk halo size was mainly related to dynamic pupil size, consistent with our previous study [22]. The disk halo size of the patients at 3 months postoperatively was also associated with the designed lenticule diameter. This is mainly because, compared with normal cornea, the edge of the lenticule may produce stray light that results in halos; thus, a larger lenticule diameter is more helpful in reducing postoperative halos.

Notably, the results of this study suggest no correlation between disk halo size after SMILE and dark pupil size. The relationship between dark pupil and vision quality after corneal refractive surgery has received much attention. Many studies have shown no relationship between the two [3-6, 23, 24], while others have shown that patients with larger dark pupils had worse postoperative visual quality [25, 26]. Our study supports the absence of a relationship and highlights the role of pupil size in dynamic pupillary light response in postoperative visual quality.

This study had certain limitations. First, because the refractive error was fully corrected with a lens before surgery, spherical aberration might have affected the evaluation of preoperative disk halo size. Second, the influence of substances, such as alcohol, on dynamic pupillary light response was not considered. Third, a long-term study of disk halo size after SMILE is necessary. It would be better to compare the outcomes with other optical quality measurements, such as OQAS and C-Quant.

In conclusion, disk halo size showed a temporary increase and returned to baseline after SMILE.

Authors' contribution Conception and design: Tian Han, Feng Zhao, Xun Chen, and Xingtao Zhou

Data collection: Tian Han, Feng Zhao, Xun Chen, Huamao Miao, and Zhuoyi Chen

Analysis and interpretation: Tian Han, Feng Zhao, Xun Chen, Huamao Miao, Zhuoyi Chen, and Xingtao Zhou

Obtained funding: Xingtao Zhou

Overall responsibility: Tian Han, Feng Zhao, Xun Chen, Huamao Miao, Zhuoyi Chen, and Xingtao Zhou

Funding information Supported in part by the National Natural Science Foundation of China for Young Scholars (Grant Nos. 
81600762 and 81700872 ), the National Natural Science Foundation of China (Grant Nos. 81570879 and 81770955), and the Project of Shanghai Science and Technology (Grant Nos. 17411950200 and 17411950201).

\section{Compliance with ethical standards}

Conflict of interest The authors declare that they have no conflict of interest.

Open Access This article is distributed under the terms of the Creative Commons Attribution 4.0 International License (http:// creativecommons.org/licenses/by/4.0/), which permits unrestricted use, distribution, and reproduction in any medium, provided you give appropriate credit to the original author(s) and the source, provide a link to the Creative Commons license, and indicate if changes were made.

\section{References}

1. Mainster MA, Turner PL (2012) Glare's causes, consequences, and clinical challenges after a century of ophthalmic study. Am J Ophthalmol 153:587-593

2. Puell MC, Perez-Carrasco MJ, Palomo-Alvarez C et al (2014) Relationship between halo size and forward light scatter. Br J Ophthalmol 98:1389-1392

3. Villa C, Gutierrez R, Jimenez JR et al (2007) Night vision disturbances after successful LASIK surgery. Br J Ophthalmol 91:10311037

4. Pop M, Payette Y (2004) Risk factors for night vision complaints after LASIK for myopia. Ophthalmology 111:3-10

5. Schallhorn S, Brown M, Venter J et al (2014) The role of the mesopic pupil on patient-reported outcomes in young patients with myopia 1 month after wavefront-guided LASIK. J Refract Surg 30: 159-165

6. Bailey MD, Mitchell GL, Dhaliwal DK et al (2003) Patient satisfaction and visual symptoms after laser in situ keratomileusis. Ophthalmology 110:1371-1378

7. Price MO, Price DA, Bucci FA Jr et al (2016) Three-year longitudinal survey comparing visual satisfaction with LASIK and contact lenses. Ophthalmology 123:1659-1666

8. Moshirfar M, McCaughey MV, Reinstein DZ et al (2015) Smallincision lenticule extraction. J Cataract Refract Surg 41:652-665

9. Li M, Zhao J, Shen Y et al (2013) Comparison of dry eye and corneal sensitivity between small incision lenticule extraction and femtosecond LASIK for myopia. PLoS One 8:e77797

10. Meiyan L, Jing Z, Huamao M et al (2014) Mild decentration measured by a Scheimpflug camera and its impact on visual quality following SMILE in the early learning curve. Invest Ophthalmol Vis Sci 55:3886-3892
11. Han T, Zheng K, Chen Y et al (2016) Four-year observation of predictability and stability of small incision lenticule extraction. BMC Ophthalmol 16:149

12. Blum M, Taubig K, Gruhn C et al (2016) Five-year results of small incision lenticule extraction (ReLEx SMILE). Br J Ophthalmol

13. Xu Y, Yang Y (2015) Small-incision lenticule extraction for myopia: results of a 12-month prospective study. Optom Vis Sci 92: 123-131

14. Miao H, He L, Shen Y et al (2014) Optical quality and intraocular scattering after femtosecond laser small incision lenticule extraction. J Refract Surg 30:296-302

15. Xu L, Wang Y, Li J et al (2015) Comparison of forward light scatter changes between SMILE, femtosecond laser-assisted LASIK, and epipolis LASIK: results of a 1-year prospective study. J Refract Surg 31:752-758

16. Guillon M, Dumbleton K, Theodoratos P et al (2016) The effects of age, refractive status, and luminance on pupil size. Optom Vis Sci 93:1093-1100

17. Puell MC, Perez-Carrasco MJ, Barrio A et al (2013) Normal values for the size of a halo produced by a glare source. J Refract Surg 29 : 618-622

18. Martucci A, Cesareo M, Napoli D et al (2014) Evaluation of pupillary response to light in patients with glaucoma: a study using computerized pupillometry. Int Ophthalmol 34:1241-1247

19. Shah R, Shah S, Sengupta S (2011) Results of small incision lenticule extraction: all-in-one femtosecond laser refractive surgery. J Cataract Refract Surg 37:127-137

20. Schallhorn SC, Kaupp SE, Tanzer DJ et al (2003) Pupil size and quality of vision after LASIK. Ophthalmology 110:1606-1614

21. Han T, Zhao J, Shen Y et al (2017) A three-year observation of corneal backscatter after small incision lenticule extraction (SMILE). J Refract Surg 33:377-382

22. Zhao F, Han T, Chen X et al (2018) Minimum pupil in pupillary response to light and myopia affect disk halo size: a cross-sectional study. BMJ Open 8:e019914

23. Myung D, Schallhorn S, Manche EE (2013) Pupil size and LASIK: a review. J Refract Surg 29:734-741

24. Haw WW, Manche EE (2001) Effect of preoperative pupil measurements on glare, halos, and visual function after photoastigmatic refractive keratectomy. J Cataract Refract Surg 27:907-916

25. Randazzo A, Nizzola F, Rossetti L et al (2005) Pharmacological management of night vision disturbances after refractive surgery results of a randomized clinical trial. J Cataract Refract Surg 31: 1764-1772

26. Alarcon A, Rubino M, Peeerez-Ocon F et al (2012) Theoretical analysis of the effect of pupil size, initial myopic level, and optical zone on quality of vision after corneal refractive surgery. J Refract Surg 28:901-906

Publisher's note Springer Nature remains neutral with regard to jurisdictional claims in published maps and institutional affiliations. 